\title{
ABORDAGEM DA ENFERMAGEM ÀS FAMÍLIAS NO ENFRENTAMENTO DO DISTANCIAMENTO SOCIAL E DO NOVO CORONAVÍRUS
}

Fernanda Lise ${ }^{1}$

Josiele de Lima Neves ${ }^{1}$

Naiane Pereira de Oliveria ${ }^{1}$

Luciana Rota Senna ${ }^{1}$

Eda Schwartz ${ }^{1}$

Lilian Moura de Lima Spagnolo1 https://orcid.org/0000-0002-1677-6140

https://orcid.org/0000-0002-8754-059X

https://orcid.org/0000-0003-3579-0535

https://orcid.org/0000-0003-4744-0372

https://orcid.org/0000-0002-5823-7858

https://orcid.org/0000-0003-2070-6177

Objetivo: Conhecer as recomendações governamentais e não governamentais para fundamentar a abordagem da Enfermagem às famílias, no enfrentamento do distanciamento social e do novo Coronavírus. Método: Trata-se de revisão narrativa da literatura, que analisou recomendações de organizações governamentais e não governamentais, nacionais e internacionais, sobre a prevenção da COVID-19 e enfrentamento do distanciamento social, apoiados no referencial teórico de enfrentamento (Coping). Resultados: Foram analisadas as recomendações de quatro organizações governamentais e duas não governamentais. A análise permitiu apresentar duas categorias denominadas “Recomendações para o enfrentamento do novo Coronavírus" e "Recomendações para o enfrentamento do isolamento social". A sintese das recomendações permitiu apresentar formas de prevenção da COVID-19 e orientações para apoiar as potencialidades das familias no enfrentamento ao distanciamento social. Conclusões: As recomendações poderão promover a abordagem segura da enfermagem, identificar os recursos das familias e construir com elas as melhores formas de enfrentamento à doença e ao isolamento social.

Descritores: Enfermagem; Família; Adaptação Psicológica; Pandemias; Quarentena.

\section{NURSING APPROACH TO FAMILIES IN THE FACING OF COVID-19 AND SOCIAL DISTANCING}

Objective: To know the national and international recommendations to support the Nursing approach to families in coping with COVID-19 and the social distance caused by the new Coronavirus. Method: This is a narrative review of the literature that analyzed national and international recommendations in governmental and non-governmental publications on the prevention of COVID-19 and social distance, supported by the theoretical framework of coping (Coping). Results: The recommendations of four governmental and two non-governmental organizations were analyzed. The analysis allowed to present two categories called "recommendations for facing novel Coronavirus" and "recommendations for facing social isolation". The synthesis of the recommendations made it possible to present ways of preventing COVID-19 and guidelines to support the potential of families in addressing social distance. Conclusions: The recommendations may promote a safe approach to nursing, identify the resources of families and build with them the best ways of coping with the disease and social isolation.

Descriptors: Nursing; Family; Adaptation Psychological; Pandemias; Quarantine.

\section{ENFOQUE DE ENFERMERÍA PARA LAS FAMILIAS EN EL ENFOQUE DEL COVID-19 Y LA DISTANCIA SOCIAL}

Objetivo: Conocer las recomendaciones nacionales e internacionales para apoyar el enfoque de Enfermeria a las familias para hacer frente a COVID-19 y la distancia social causada por el nuevo coronavirus. Método: Esta es una revisión narrativa de la literatura que analizó las recomendaciones nacionales e internacionales en publicaciones gubernamentales y no gubernamentales sobre la prevención de nuevo coronavirus y la distancia social, con el apoyo del marco teórico de afrontamiento (afrontamiento). Resultados: se analizaron las recomendaciones de cuatro organizaciones gubernamentales y dos no gubernamentales. El análisis permitió presentar dos categorías llamadas "recomendaciones para enfrentar COVID-19" y "recomendaciones para enfrentar el aislamiento social". La sintesis de las recomendaciones permitió presentar formas de prevenir COVID-19 y pautas para apoyar el potencial de las familias para abordar la distancia social. Conclusiones: Las recomendaciones pueden promover un enfoque seguro de enfermería, identificar los recursos de las familias y construir con ellos las mejores formas de hacer frente a la enfermedad y el aislamiento social.

Descriptores: Enfermería; família; Adaptación Psicológica; Pandemias; Cuarentena.

UUniversidade Federal de Pelotas - UFPel, RS.

Autor Correspondente: Fernanda Lise E-mail: fernandalise@gmail.com

Recebido em 10/5/2020 Aceito: 06/06/2020 


\section{INTRODUÇÃO}

A crise global ocasionada pela pandemia do novo Coronavírus (SARS-CoV-2) destacou a Enfermagem como uma das profissões essenciais no atendimento ao paciente hospitalizado, bem como seu papel preponderante na prevenção da infecção, no manejo das emoções e na promoção do enfrentamento saudável ${ }^{1}$.

Nesse sentido, um dos desafios está relacionado à transmissibilidade do novo coronavírus (SARS-CoV-2), a qual pode ser maior que a transmissão da Síndrome Respiratória Aguda Grave ou pandêmica (SARS-CoV) e à Síndrome Respiratória no Oriente Médio (MERS-CoV), ocorridas no passado. Isso impôs a necessidade de isolamento social dos indivíduos, com a quarentena, para evitar a contaminação em massa e, por consequência a sobrecarga no atendimento do sistema de saúde ${ }^{2}$.

A quarentena, caracterizada como uma medida administrativa de restrição por até 40 dias ou mais de reclusão, aplicado às pessoas sadias ou que podem ter sido contaminadas por alguma doença, a fim de reduzir a transmissibilidade. O isolamento social, por sua vez, está relacionado ao confinamento de uma pessoa sintomática ou em investigação clínica e laboratorial por 14 dias ou mais, até que finalize o período de transmissibilidade ${ }^{3}$. Nesse estudo, ambos os termos serão utilizados sem distinção, dada as semelhanças de ambos, no enfrentamento à crise da pandemia de COVID-19.

O confinamento pode desencadear sentimentos estressantes que impactam diretamente na vida dos indivíduos, os quais podem ser exacerbados, em decorrência da abrupta mudança na rotina e dos sentimentos experimentados durante esse período, que vão desde incerteza, estigmatização, ansiedade, insônia, raiva, solidão, frustração, preocupação, separação, até a prática de suicídio ${ }^{4-5}$

Para lidar com estes fatores, os individuos desenvolvem um conjunto de estratégias de cunho cognitivo-comportamental ${ }^{6}$. O Modelo Coping propõe que o enfrentamento seja dividido em duas categorias ${ }^{7}$. A partir da avaliação dos eventos, o indivíduo busca estratégias de enfrentamento, através de esforços podem ter duas funções principais: planos voltados a resolução ou alteração do evento estressor, vistas como dispositivos de adaptação, chamado de Coping focado no problema e a estratégia que visa organizar a resposta emocional que o fator ou evento estressante causa ao indivíduo, chamada de Coping focado na emoção ${ }^{8}$.

Portanto, durante o cenário atual, é necessário o reconhecimento de que em alguns aspectos, relacionados à infecção pelo novo coronavirus, algumas pessoas terão mais controle da situação e outras não serão capazes de instaurar o mesmo controle, em virtude da variabilidade do contexto em que cada indivíduo se insere ${ }^{9}$. Diante da crise global na saúde, é fundamental o papel da Enfermagem no enfrentamento da pandemia gerada pelo novo coronavírus (SARS-CoV-2), em todas as áreas de atuação.

Esse estudo objetivou conhecer as recomendações governamentais e não governamentais, para fundamentar a abordagem da Enfermagem às famílias no enfrentamento do distanciamento social e do novo coronavírus.

\section{MÉTODO}

\section{Tipo de estudo}

Trata-se de um estudo de revisão narrativa da literatura, desenvolvida a partir da necessidade de reunir informações de documentos e pesquisas, com diferentes abordagens, para uma síntese atualizada das orientações e conhecimento ${ }^{10}$. O desenvolvimento envolveu três etapas: a primeira, relacionada a contextualização da experiência; a segunda foi a busca por literatura relevante; e a terceira, a análise das fontes documentais finais e sintese dos resultados.

A contextualização da experiência está relacionada à necessidade de conhecer, em curto espaço de tempo, as recomendações nacionais e internacionais para a prevenção da COVID-19, para apoiar a abordagem da Enfermagem no enfrentamento do isolamento social experienciado pelas famílias, em decorrência da pandemia pelo novo coronavírus (SARS-CoV-2).

\section{Coleta dos dados}

A busca pela literatura foi realizada por quatro revisores, a partir da consulta em websites governamentais e não governamentais, nacionais e internacionais, selecionados por sua reconhecida importância internacional na apresentação de diretrizes para profissionais, na atenção à saúde das famílias. Foram consultados os websites governamentais do Ministério da Saúde - Brasill1; do National Health Service United Kingdoml'2; do Center of Diseases Control and Prevention (CDC) do governo Norte Americano ${ }^{13}$ e do Department for Children, Schools and Families ${ }^{14}$. E dos websites não governamentais da Organização Mundial da Saúde (OMS) ${ }^{14} \mathrm{e}$ da International Family Nursing Association IFNA ${ }^{4}$.

\section{Análise dos dados}

A análise se deu a partir da leitura e síntese das recomendações das fontes consultadas, como estratégias para aprimorar a abordagem da Enfermagem com famílias, no enfrentamento do distanciamento social e do novo coronavírus, e apoiadas nos conceitos do Modelo de Coping? Essa perspectiva sugere que o indivíduo busca estratégias para o enfrentamento, através de esforços, assumindo duas funções principais: 1) planos voltados a resolução ou alteração do evento estressor, chamado de Coping focado no 
problema - nesse estudo, a infecção pelo novo coronavírus; e 2) estratégia que visa organizar a resposta emocional que o fator ou evento estressante causa ao individuo, chamada de Coping focalizado na emoção - nesse estudo, o isolamento social ${ }^{8}$.

\section{RESULTADOS}

A sintese das recomendações das seis organizações, sendo, quatro organizações governamentais ${ }^{11-14}$ e duas não governamentais ${ }^{4,15}$, permitiram apresentar duas categorias. A primeira categoria denominada "Coping focado no problema: Recomendações para o enfrentamento do novo coronavírus" com recomendações ${ }^{11-15}$ apresentadas no quadro 1. A segunda categoria denominada "Coping focado nas emoções: Recomendações para o enfrentamento do isolamento social", com recomendações ${ }^{4.14,15}$ apresentadas no quadro 2.

Quadro 1 - Recomendações para o enfrentamento da infecção pelo novo coronavírus, 2020.

\section{Ministério da Saúde ${ }^{11}$}

Lave as mãos até altura dos punhos com água e sabão ou higienize as mãos com álcool em gel 70\%;

Cubra nariz ou boca ao tossir ou espirrar e evite tocar olhos, nariz e boca sem lavar as mãos;

Mantenha uma distância de 2 metros de qualquer pessoa ao tossir ou espirrando;

Evite abraço, beijos e apertos de mão;

Higienize celular e brinquedos de crianças;

Não compartilhe objetos de uso pessoal como: talheres, toalhas, pratos e copos;

Evite aglomerações:

Durma bem, tenha uma alimentação saudável e faça uma atividade física.

Mantenha o ambiente limpo e ventilado;

Sempre utilize máscaras (artesanais ou caseiras de tecido) ao sair de casa;

Caso seja inevitável uma viagem, siga as recomendações de saúde local;

Caso retorne de um país internacional, coloque-se em isolamento voluntário por sete dias, mesmo sem apresentar sintomatologia;

Caso retorne de viagens locais, atentar-se às condições de saúde pelo período de 14 dias;

Em caso de doença, isolar-se de todos os moradores da mesma residência, mantenha utensilios pessoais separados, lixo produzido deve ser separado e descartado, os outros moradores devem adotar o isolamento por 14 dias, manter a casa limpa com água sanitária ou álcool 70\% e ventilada;

Caso apresente sintomas com alteração na respiração, procure orientação médica.

\section{National Health Service ${ }^{12}$}

Ficar em casa é a melhor maneira de evitar a propagação do vírus;

Sair de casa apenas para casos limitados, como: comprar alimentos e medicamentos, em menor frequência possivel, realizar uma forma de exercício por dia, garantindo uma distância mínima de 2 metros de qualquer pessoa, qualquer necessidade médica, viajar por fins profissionais que não podem ser realizados em domicílio; 
Lavar as mãos com água e sabão durante 20 segundos;

Usar gel desinfetante caso água e sabão não estejam disponíveis;

Lavar as mãos assim que chegar em casa;

Cobrir boca e nariz com lenço de papel ou manga de roupas ao tossir e espirrar, descartar o lenço de papel imediatamente após o uso;

Não tocar olhos, nariz e boca sem que as mãos estejas limpas e/ou desinfetadas;

Em caso de pessoas do grupo de risco ou com algum sintoma da doença, colocar-se em auto isolamento.

Center of Diseases Control and Prevention ${ }^{13}$

Monitorar sintomas (febre, tosse e dificuldades para respirar). Procure o serviço de saúde. Atenção a sintomas como dor torácica, boca e/ou face cianótica;

Usar máscara e etiqueta respiratória (se tossir ou espirrar, cobrir a boca e o nariz com um lenço de papel), despreze os papéis usados em lixeira forrada;

Evitar tocar nos olhos, nariz e boca com as mãos sujas;

Lavar as mãos por pelo menos 20 segundos;

Usar álcool em gel 60\%, se não for possivel usar água e sabão para desinfetar as mãos;

Se estiver doente, não compartilhe utensilios domésticos; limpe o ambiente, superfícies (telefones, controles remotos, balcões, mesas, maçanetas, utensílios de banheiro, teclados, tablets e mesas de cabeceira) e banheiro diariamente;

Manter distanciamento social.

\section{Organização Mundial da Saúde ${ }^{15}$}

Lavar as mãos regularmente com água e sabão, quando não for possível limpá-las com álcool 70;

Manter no mínimo um metro de distância de pessoas que apresentam sintomas como tosse e/ou espirro;

Evitar tocar o rosto, proteja boca e nariz ao espirrar ou tossir;

Caso apresente algum sintoma, manter-se em casa;

Evitar atividades prejudiciais ao pulmão;

Evitar viagens desnecessárias e manter-se afastado de aglomerações;

Praticar o distanciamento físico. 
Quadro 2 - Recomendações para o enfrentamento do isolamento social, 2020.

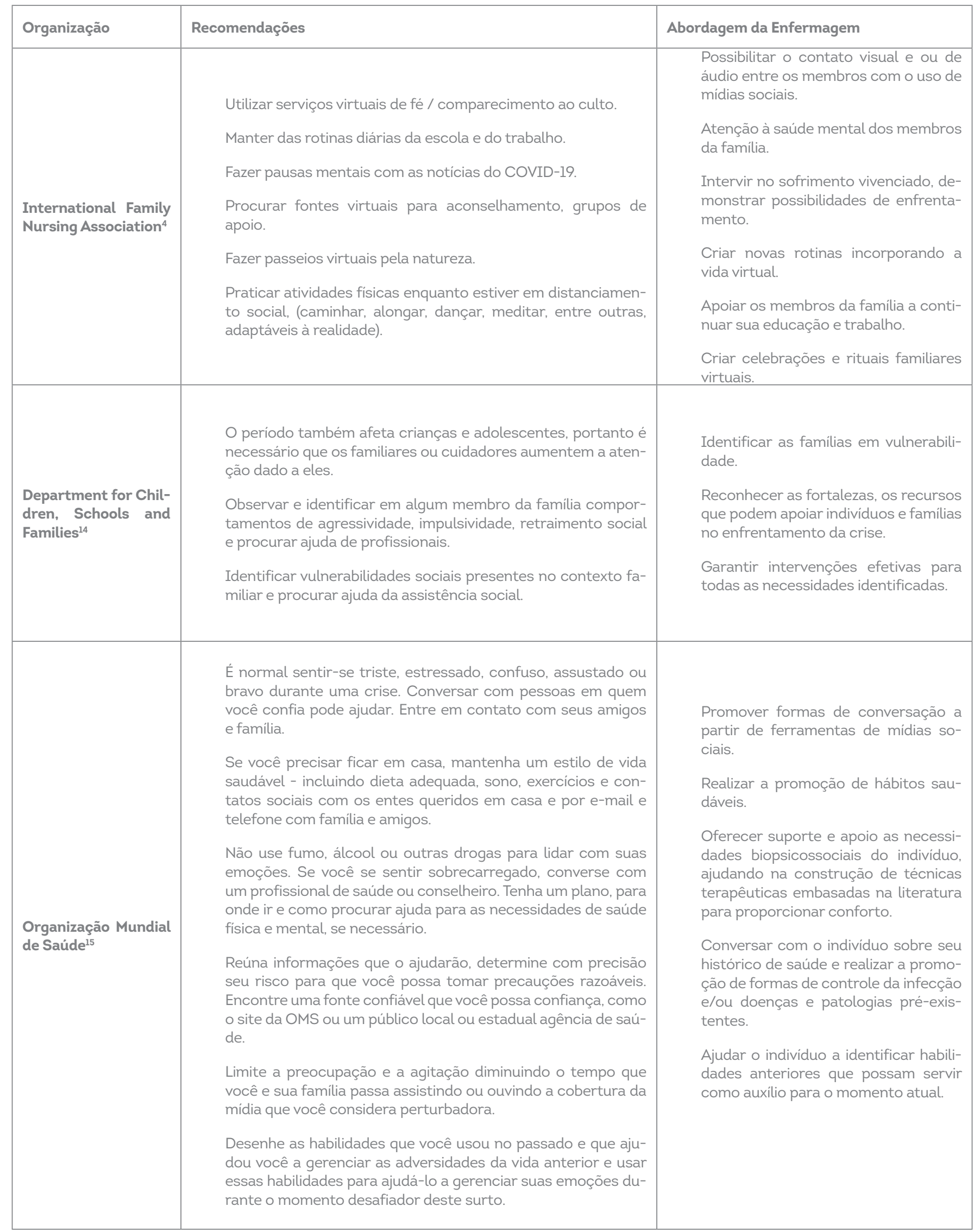




\section{DISCUSSÃO}

Coping focado no problema: Recomendações para o enfrentamento do novo coronavirus

Após a recente crise causada pelo novo coronavirus, as recomendações governamentais e não governamentais ${ }^{4,11-15}$, corroboram com o modelo de Coping, apontando a necessidade de abordagem imediata para resolver o problema causado pelo novo coronavírus na prevenção da COVID-19. Desde o primeiro registro, em dezembro de 2019, na cidade de Wuhan, na China, o novo coronavirus (SARS-CoV-2), ou COVID-19, como foi denominada a doença respiratória infecciosa, foi considerada uma pandemia pela Organização Mundial da Saúde ${ }^{2}$ após atingir todos os continentes. Sua transmissão pelo contato direto de uma pessoa saudável com gotículas, aerossóis e fezes de um portador da COVID-19 ou superfícies contaminadas. As experiências da China, Alemanha e de Singapura mostram que uma pessoa contaminada pode iniciar a transmissão a partir de 24 horas após contaminar-se até três ou quatro semanas após a manifestação dos sintomas. Em decorrência disso, estabeleceu-se a recomendação para manter distanciamento de um metro entre as pessoas e isolamento social de 14 dias se a pessoa apresentar algum dos sintomas de hipertermia, tosse, odinofagia e ou dispnéia, bem como, se tiver histórico de viagem e ou contato com alguém que esteve no exterior ${ }^{16}$.

As recomendações nacionais e internacionais ${ }^{4.11-15}$ e os resultados de experiências recentes com o novo coronavírus (SARS-CoV-2) estão ligadas à prevenção e ao tratamento. A prevenção do contágio do novo coronavírus (SARS-CoV-2) assemelha-se as infecções do trato respiratório, que incluem lavar as mãos com água e sabão, uso de álcool gel e etiqueta respiratória. Buscar atendimento médico quando apresentar febre, tosse e dificuldade para respirar e relatar caso tenha histórico de viagens ${ }^{11,15}$. Essas recomendações requerem intervenções para a manutenção do bem-estar das famílias.

Pode-se perceber algumas discrepâncias entre as medidas adotadas por diferentes países, como por exemplo, no Reino Unido ${ }^{12}$, dentre as exceções do isolamento, pode-se sair de casa para a prática de exercícios físicos, o que não é recomendado por países como Brasill ${ }^{11}$ e Estados Unidos ${ }^{13}$. De acordo com o Centro de Controle e Prevenção de doenças dos Estados Unidos ${ }^{13}$, preconiza-se o uso de álcool $60 \%$ quando água e sabão não estiverem disponiveis para a higienização das mãos. Nos demais países e na Organização Mundial de Saúde opta-se pelo álcool 70\%11,12,15

No Brasil, a testagem da contaminação pelo novo coronavirus (SARS-CoV-2) está sendo realizada em profissionais de serviços de saúde e segurança, bem como, pacientes em estado grave e óbitos relacionados a problemas respiratórios ${ }^{11}$.
Portanto as recomendações para pacientes que apresentem sintomatologia leve, é de se colocarem em um auto isolamento, uso de máscaras faciais, etiquetas de higiene respiratória e das mãos para evitar a disseminação do vírus.

Um consenso percebido entre as organizações de saúde do Brasill ${ }^{11}$ e dos Estados Unidos ${ }^{13}$ é a promoção da limpeza dos espaços domiciliares, como pisos, cadeiras, mesas, maçanetas, entre outros. No Brasil, a especificação é para que esta limpeza seja feita com água sanitária ou álcool 70\% e priorize a ventilação dos ambientes ${ }^{11}$. Nos Estados Unidos, a recomendação é para a utilização de água, sabão ou outros detergentes, atentando-se para os rótulos e instruções dos produtos $^{13}$

No Brasil, nos Estados Unidos e no Reino Unido, de acordo com as atualizações da Organização Mundial de Saúde, todos os individuos ao sairem de casa deve-se utilizar máscaras faciais artesanais e/ou caseiras, posto que, dada a carência, a utilização de máscaras industrializadas deve ser restrita a profissionais de saúde e socorristas ${ }^{11-13.15}$.

Para tanto, o isolamento social ou quarentena, é uma medida de emergência pública tomada para evitar a propagação do vírus, que ainda não tem suas etiologias e abordagens bem definidas. Diante disso, a Enfermagem tem competências para usar as recomendações no desenvolvimento de estratégias de prevenção de infecção pelo novo coronavírus e promoção da saúde das famílias.

Coping focalizado na emoção: Recomendações para o enfrentamento do isolamento social

Estudos prévios contribuem para destacar a importância do distanciamento social, pois, reflete significativamente na coletividade. Embora a motivação primária seja a própria proteção, a vida de profissionais da área da saúde pode ser menos afetada, já que o número de pessoas infectadas tende a ser menor. Além disso, reduz o impacto emocional em pessoas hospitalizadas, em quarentena, e ou na expectativa de terem se infectado com o vírus. Neste contexto, os órgãos governamentais podem intensificar a comunicação clara, bem compreendida, afim de conscientizar, informar, educar e tranquilizar a população durante períodos críticos como os de distanciamento social obrigatório.

A partir da experiência chinesa, as intervenções recomendadas, como resposta à crise causada pelo novo coronavírus (SARS-CoV-2), incluíram a abordagem ao medo da doença e as dificuldades de adaptação. As propostas de médicos, psicólogos e psiquiatras incluíram manter contato telefônico com os indivíduos em isolamento, usar aplicativos e informações em website para orientar sobre os cuidados, e oferecer suporte psicossocial as pessoas na quarentena ${ }^{17}$. 
Para tanto, a abordagem da Enfermagem às famílias ao enfrentamento do isolamento social requer o uso das competências para melhorar e promover a saúde das famílias ${ }^{18}$. Apoiado em pesquisas que mostram a importância dos enfermeiros e como podem ajudar famílias que são isoladas ou separadas nesses tempos difíceis, a IFNA listou ações capazes de promover serenidade, união, tranquilizar, encorajar e estimular a afetividade na abordagem com familias, as quais foram adaptadas e apresentadas a seguir ${ }^{4}$.

Dentre as recomendações, destacam-se possibilitar a aproximação dos membros familiares, com o uso de mídias sociais, cria-se oportunidades para diminuir a solidão desencadeada pela separação física e gerar sensação de tranquilidade a partir da capacidade da família em fortalecer-se, mesmo que fisicamente distante, reforça-se a espiritualidade, na sensação de pertencimento proporcionado pelo contato visual e ou auditivo com entes queridos ${ }^{4}$.

Durante o enfrentamento, a estigmatização decorrente da doença ameaçadora e a incertezas sobre a evolução da pandemia e tempo de reclusão podem ter consequências à saúde mental de todos os membros da família, pela capacidade de atuar como um gatilho para desencadear desequilibrios emocionais, por isso, focar no bem-estar, esclarecimentos das dúvidas com informações claras e precisas pode diminuir o preconceito e a insegurança ${ }^{4}$.

As intervenções de Enfermagem no enfrentamento ao sofrimento gerado pela doença incluem apoio na adaptação as novas formas de desenvolver as atividades da escola ou do trabalho à distância ou virtuais. Reconhecer os esforços nesse processo é fundamental para o sucesso do ajuste a nova realidade, reforçando a importância da continuidade das atividades para o sentimento de realização e redução da frustração ${ }^{4}$.

Diante da preocupação e até mesmo do pânico gerado pela avalanche de informações nos meios de comunicação em massa, muitas vezes com notícias impactantes e constantes ao longo do dia, as quais podem ter consequências negativas aos indivíduos, por desencadear insônia e ansiedade. Por isso, orientar fazer pausas mentais das notícias do COVID-19 podem impactar positivamente. Assim como orientar a busca de grupo de apoio em redes sociais virtuais, assim como criar celebrações e rituais familiares, visitar museus e parques pode reduzir a inquietude e promover o afeto mútuo ${ }^{4}$.

Durante o isolamento social, a prática de atividades físicas pode ser adaptada as condições das famílias em casa, tal como, caminhar, alongar, dançar, as quais podem ser estimuladas, por exemplo, com vídeos online. A manutenção dos exercícios físicos é importante para aumentar a imunidade, reduzir a tensão muscular, combater o estresse e promover o bem-estar físico e mental.
Em complemento, a OMS reafirma a importância dos hábitos que preservem a saúde mental do individuo ${ }^{15}$, para os quais a Enfermagem tem competências para intervir com o uso de técnicas de relaxamento como alongamento, controle da respiração e meditação, as quais têm suas eficácias comprovadas cientificamente e fazem parte da rotina profissional no cuidado direto às famílias ${ }^{15}$.

Na abordagem prática com famílias, faz-se necessário identificar as famílias que em risco e intervir com rapidez. Focar a atenção nas famílias em vulnerabilidade socioeconômicas; famílias com baixa escolaridade, doenças crônicas e ou incapacitantes para atender as necessidades levantadas e fortalecer as capacidades dos membros em apoiar e cuidar uns dos outros ${ }^{14}$

Outro fator estressante durante o período do isolamento social é a preocupação com questões de saúde, como doenças pré-existente, pessoas em grupo de risco que incluem idosos, hipertensos, diabéticos, mulheres em período puerperal, pessoas em recuperações cirúrgicas. A Enfermagem pode oferecer cuidados seguros a estes indivíduos para auxilia-los a identificar alterações corporais, uso correto das medicações, por meio de consultas virtuais, e recomendar, quando houver necessidade, serviço de atendimento presencial, além de estimular o autocuidado.

\section{Limitações do estudo}

As possiveis limitações do estudo podem estar relacionadas às alterações nas recomendações, as quais poderão ser aprimoradas e ou adequadas de acordo com a evolução da doença ou mudanças sociais. Porém, isso não impede afirmar que as abordagens apresentadas estão apoiadas nos conceitos do enfrentamento de coping, em recomendações de reconhecidas organizações governamentais e não governamentais, nacionais e internacionais, capaz de oferecer diretrizes para profissionais da área da saúde.

\section{Contribuições para a prática}

A abordagem da Enfermagem pode facilitar a adaptação, assegurar que os cuidados sejam realizados para promover a desestigmatização, a continuidade das atividades seguras à saúde, a prática de atividades físicas, a manutenção do equilibrio emocional, estimular o afeto e a união entre os indivíduos e suas famílias para o enfretamento saudável do isolamento social causado pela pandemia.

\section{CONCLUSÃO}

As recomendações das organizações governamentais e não governamentais poderão oferecer à Enfermagem subsídios para a abordagem segura no uso de suas competências e na prática com famílias promover estratégias de intervenções 
seguras, facilitar a transição no processo de adaptação, identificar as potencialidades das famílias e proporcionar bem-estar no enfretamento à doença e ao isolamento social.

Contribuição dos Autores: FL: planejamento, execução, redação e revisão final do artigo; JLN: planejamento, execução, redação, revisão final do artigo; NPO: planejamento, execução, redação, revisão final do artigo; LRS: planejamento, execução, redação, revisão final do artigo; ES: planejamento, redação, revisão crítica e final do artigo; LMLS: planejamento, redação, revisão crítica e final do artigo.

Agradecimentos: Ao Conselho Nacional de Desenvolvimento Científico e Tecnológico (CNPq) pela bolsa de produtividade em pesquisa do quinto autor.

\section{REFERÊNCIAS}

1. World Health Organization. State of the World's Nursing Report - 2020 [Internet]. 2020 [cited 2020 abr 21]. Avaliable from: https:// www.who.int/publications-detail/nursing-report-2020.

2. World Health Organization. O\&A on coronaviruses. [Internet]. 2020 [cited $2020 \mathrm{abr} 21$ ]. Avaliable from: https://www.who.int/newsroom/q-a-detail/q-acoronaviruses. 2020

3. Agência Brasil. Covid-19 saiba a diferença entre quarentena e isolamento [Internet]. 2020 [cited 2020 abr 21 ]. Avaliable from: https:// agenciabrasil.ebc.com.br/saude/noticia/2020-03/covid-19-saiba-diferenca-entre-quarentena-e-isolamento.

4. International Family Nursing Association (IFNA). COVID-19 Pandemic and Family Nursing: IFNA President and President-Elect Offer a Message to Members [Internet]. 2020 [cited 2020 abr 21]. Avaliable from: https://internationalfamilynursing.org/2020/03/27/covid19-pandemic-ifna-president-and-ifna-president-elect-offer-a-message-to-members/.

5. Brooks SK, Webster RK, Smith LE, Woodland L, Wessely S, Greenberg N et al. The psychological impact of quarantine and how to reduce it: Rapid review of the evidence. The Lancet. [Internet]. 2020 [cited 2020 abr 21];395(10227):912-920. Avaliable from: https://doi. org/10.1016/S0140-6736(20)30460-8.

6. Folkman S, Moskowitz JT. Coping: Pitfalls and Promise. Annu. Rev. Psychol. [Internet]. 2004 [cited 2020 abr 21];55:745-74. Avaliable from: https://doi.org/10.1146/annurev.psych.55.090902.141456.

7. Folkman S, Lazarus RS. An analysis of coping in a middle-aged community sample. J. Health Soc. Behav. [Internet]. 1980 [cited 2020 abr 21]:21:219-239. Avaliable from: https://doi.org/10.2307/2136617.

8. Folkman S. Stress, coping, and hope. Psycho-Oncology. [Internet]. 2010[cited 2020 abr 21]; 19(9):901-908. Avaliable from: https://doi. org/10.1002/pon.1836.

9. Mind Garden. Tools for the positive transformation. Coping through the Coronavirus. [Internet]. 2020. [cited 2020 abr 21]. Avaliable from: https://www.mindgarden.com/blog/post/50-coping-through-the-coronavirus?utm_source=SLNL-0-3207338-3447928-Eutm_ medium=email\&utm_campaign=SLNL-0-3207338-3447928-

10. Grupo Anima. Manual revisão bibliográfica sistemática integrativa: a pesquisa baseada em evidências. [Internet]. 2014 [cited 2020 mar 20]. Avaliable from: http://biblioteca.cofen.gov.br/wp-content/uploads/2019/06/manual_revisao_bibliografica-sistematicaintegrativa.pdf.

11. Ministério da Saúde (BR). Protocolo de Manejo Clínico para o Novo Coronavírus (2019-nCoV). [Internet]. 2020. [cited 2020 mar 25]. Avaliable from: https://portalarquivos2.saude.gov.br/images/pdf/2020/fevereiro/ll/protocolo-manejo-coronavirus-FINAL.pdf.

12. National Health Service (UK). Advice for everyone. Coronavirus (COVID-19). [Internet]. 2020. [cited 2020 mar 25]. Avaliable from: https://www.nhs.uk/conditions/coronavirus-covid-19/.

13. Centers for Disease Control and Prevention. Coronavirus disease 2019 (COVID-19). [Internet]. 2020. [cited 2020 mar 25]. Avaliable from: https://www.cdc.gov/coronavirus/2019-ncov/index.html.

14. United Kingdom Government. Department for Children, Schools and Families. Think Family Toolkit: Improving support for families at risk Strategic overview. [Internet]. 2009. [cited 2020 mar 25]. Avaliable from: https://webarchive.nationalarchives.gov.uk/20130323053534/ https://www.education.gov.uk/publications/eOrderingDownload/Think-Family.pdf

15. World Health Organization (WHO). Novel coronavirus (2019-nCoV) situation reports. [Internet]. 2020. [cited 2020 mar 25]. Avaliable from: https://www.who.int/emergencies/diseases/novel-coronavirus-2019/situation-reports/.

16. Organização Pan-Americana de Saúde (OPAS). Folha informativa - COVID-19 (doença causada pelo novo coronavirus). [Internet]. 2020. [cited 2020 abr 04]. Avaliable from: https://www.paho.org/bra/index.php?option=com_ content\&view=article\&id=6101:covidl9\&Itemid=875.

17. Zhang J, Wu W, Zhao X, Zhang W. Recommended psychological crisis intervention response to the 2019 novel coronavirus pneumonia outbreak in China: a model of West China Hospital. Precision Clinical Medicine. [Internet]. 2020. [cited 2020 abr 04]:3(1):3-8. Avaliable from: https://academic.oup.com/pcm/article/3/1/3/5739969.

18. International Family Nursing Association (IFNA). Declaração de posição sobre competências do enfermeiro generalista na prática de cuidados à familia. [Internet]. 2015. [cited 2020 abr 04]. Avaliable from: https://internationalfamilynursing.org/wordpress/wp-content/ uploads/2015/07/GC-PDF-document-Portuguese-language-translation.pdf. 